resources and the inveteracy of the prejudices in which we are still immersed.

We, Sir, of the College of Physicians, have now entered upon the 389 th year of our corporate existence; we took part in your foundation, as we fostered the rising of the Royal Society 150 years before you; and in the plentitude of our priority and precedence, our wide influence acquired by the promotion of natural knowledge, we infer the assurance of your progression from the solid foundations on which you are building and from the eminent services you have already rendered. Your progress will not slacken; discovery will beget discovery ; practical work, prudent and sagacious, will regulate the scope of generalisation, and knowledge will overflow-to the benefit of mankind, the credit of our race, and the honour of the Geological Society of London.

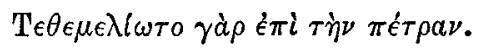

\section{A NOTE ON A RECENT EPIDEMIC OF TRYPANOSOMIASIS AT MAURITIUS.}

BY ALEXANDER EDINGTON, M.D. EDIN., D.P.H. EDIN. \& GLASG., F.R.S. FDIN.,

LATE DIRECTOR OF THE GOVERNMENT BACTERIOLOGICAL INSTITUYE, GRAHAMSTOWN, CAPE COLONY;

AND

JOHN MORTON COUTTS, M.D. LOND., D.P H. CAMB.,

LATE SENIOR ASSTSTANT AT THE GOVERNMENT BACTERIOLOGIOAT INSTITUTE, GRAHAMSTOWN, CAPE COLONY.

IN recent years no disease, in its relation to man and animals, has been productive of greater interest than trypanosomiasis. Apart from the fact that epidemics among animals have occurred in countries widely separate and that it has seldom been possible to point to the exact line by which infection has arrived, the determination that one species at least affects man, and, in general, the intractable order of the malady have sufficed to stimulate persistent investigation.

A brief consideration of the recent epidemic at Mauritius among the domesticated animals will suffice to show the danger to be apprehended from such an occurrence in its relation to the public finances and the interference with commerce, and also the more or less unsatisfactory outcome of scientific investigation.

The Island of Mauritius is mainly concerned, commercially, in the growth of sugar-cane, of which the collection is mostly made by Indian coolies, while cattle are used to a very great extent as the means of transport. About the end of the year 1901 a disease had made its appearance among the cattle, later among mules, and gradually extended, so that by the end of July, 1902, when one of us (A. E.) arrived at Mauritius to inquire into it the whole island was already infected. By this time, as is obvious, any attempt to deal with the epidemic by the slaughter of infected animals was impracticable, and the only course open was to safeguard, as far as possible, such animals as appeared healthy and to attempt to cure the sick. Among infected mules death was the rule; of the cattle infected considerable numbers made recovery, but the deaths, nevertheless, were so great as to impede the collection of the sugar canes.

Regarding the origin of the epidemic two views were open to consideration: (1) its importation from India; and (2) its previous existence in the island. The importation of cattle for Mauritius was ordinarily made from Madagascar, but towards the end of the Boer war, possibly owing to the heavy demands which had been made on Madagascar for cattle, there was a great difficulty in obtaining supplies for Mauritius. Under such circumstances the suggestion was made and acted upon to import some cattle from India. The first lot of ten animals were received at Mauritius on July 18ch, 1901. One died from injuries, while the remainder went to one estate. No disease occurred on this place until nine months afterwards, at which time the island was already well infected. It is, how ever, to the second cargo to which suspicion pointed. This curgo arrived in September, 1901, and, according to the information obtained, no deaths occurred on board and all animals arrived in splendid condition. Many were sold in small lots, but it is interesting to follow up two big batches of 50 each which went to different areas. Of one lot which went to the district of Palma, 18 died during the latter part of November, 1901 of the other 50 which went to Mare Seche, one animal died during a cyclone on Dec. 5th but no other death occurred until Jan. 7th, 1902. By May 14th, by which time the whole island was infected, seven of these Indian cattle had died and nine Madagascar animals. In the course of this inquiry, conducted with the personal assistance of the chief of the health department, veterinary surgeons, and representative planters, the history of every Indian animal which had been imported was followed up.

With reference to the suggestion that the malady had been already on the island the fact was elicited that a disease had been formerly known to occur in Mauritius among mules, and that the symptoms found during life and after death had had striking resemblances to those of the epidemic disease. Among those who affirmed this were medical men of the health department, veterinary surgeons, and planters. The investigator therefore reported ${ }^{1}$ that while the evidence failed to establish the responsibility of the Indian cattle it tended to show that a disease similar to trypanosomiasis had already been in existence (at a former time) on the island, and he suggested that inquiry should be made in the adjacent islands to determine whether any similar disease or any suspicious cases had been found. Since then information has been acquired showing that a case of trypanoso. miasis had actually been found in a cow on the adjacent French island of Reunion by Dr. Vassal of the Pasteur Institute in August, 1901, a period which antedates the appearance of the epidemic in Mauritius. Trypanosomes were found in great abundance in the blood, spleen, and kidneys, and although Dr. Vassal's attention was given to the matter he failed to find any other case on the island. Commenting on this, Laveran and Mesnil ${ }^{2}$ say that the origin of the occurrence is the more inexplicable since the infected animal was one which had been born on the island. This island is about 100 miles distant from Mauritius.

This difference of opinion regarding the origin of the disease at Mauritius recalls the epidemic of trypanosomiasis at the Philippines. In that case Salmon and Stiles thought it to be a recent importation, but medical men and veterinary surgeons there maintain that a disease known as "calentura" formerly existed which was identical with the epidemic malady.

The African tsetse fly is known to be the carrier in infected areas of that continent of the trypanosome known as trypanosoma Brucei, but in Mauritius there is no such insect. On the other hand, a species of stomoxys is present in enormous numbers, and its biting powers and propensities are made painfully evident to anyone who comes near the cattle on the plantations By the kindness of Professor (now Sir E.) Ray Lankester we have had an authoritative opinion on the species sent to him by us. Mr. E. E. Austen reports as follows: "Stomoxys nigra Marquart, a species originally described from Reunion but widely distributed in the Ethiopian regions, since, as shown by the series in our collection, it is found right across Africa from the Gambia eastward." In Mauritius this fly is called the "mouche bœuf." There was no difficulty whatever in finding enormous numbers of trypanosomes in the stomachs of these insects. Indeed, many of the film preparations made from the stomach contents were swarming with parasites. M. Daruty de Grandpré, curator of the museum at Mauritius, sncceeded in infecting a healtby dog to which he applied eight flies. It has not hitberto been believed that stomoxys could be a carrier of trypanosomes, but the imputation conveyed by observation and experiment at Mauritius at the least calls for this point being investigated under circumstances where possible fallacy can be excluded.

Compared with the trypanosoma Brucei the Mauritios trypanosome seems smaller. If, however, the flagellum is included the lengths are nearly alike. The body of the Mauritius form is, however, somewhat shorter, and the flagellum longer than obtains in the trypanosoma Brucei. In the blood of animals in which either the tsetse or the Mauritius disease runs a chronic course, particularly in the rabbit, we have found on many occasions towards the latter period of the maladies irregular protoplasmic masses which agree with several described by Bradford and Plimmer $^{3}$ in

1 Report to the Governor of Mauritius, 1902.

2 Try panosomes et Trypanosomiases.

3 The Trypanosoma Brucei, the Organism found in Nagana or Tretse-

Quarterly Journal of Microscopical Science, vol. xlv., N.S. 
their account of the morphology of the trypanosoma Brucei. Under conditions which may by analogy be considered as unfavourable to the life of the parasites, peculiar forms may be observed. These are readily seen in blood which has been kept for some time after withdrawal or when other serum has been added to it. In such cases there is a tendency in some parasites to adbere by their blunt or posterior extremities. Where a number are concerned a veritable rosette may be formed. In fresh blood, however, it is not unusual to find examples. In the blood films of cattle running on the estates, which were made directly on the spot, not only were they common, but often two or three examples might be seen on the one fiald under observation. Bradford and Plimmer consider these as a manifestation of "conjugation," while Laveran and Mesnil qualify it as only occurring nader abnormal conditions.

On Dr. Elington's return to South Africa from Mauritius he brought with him several guinea-pigs and a dog already infected with the Mauritius disease. One of us (J. M. C.), arriving in South Africa at a later date, carried with him by the kindness of Professor John Rose Bradford, several guinea-pigs which had been infected at the Brown Institution, London, with tsetse-fly disease. The arrival of these animals at the Bacteriological Institute, Grahamstown, per mitted experimental work to be undertaken, the results of which may be briefly alluded to.

Experimental Work with the Mauritius Trypanosomes.

$\operatorname{Dog} N_{0}$. 1.-The animal was inoculated intravenously with one cubic centimetre of blood from an infected dog. Trypanosomes appeared in the blood on the seventh day on the tenth day the blood was swarming with them. After five days they disappeared, but after a further period of three days reappeared and continued in great numbers until death, which occurred 35 days after inoculation Usually the first appearance of trypanosomes in the blood is associated with a rise of temperature in the animals, but as the temperature curve attains its highest altitude for that particular febrile period the parasites begin to disappear and the temperature falls. After the fall trypanosomes again make their appearance and the fever recrudesces. The regularity of these phenomena suggests that high fever is inimical to the vitality of the parasites in the blood. Post-mortem examination of the dog revealed marked enlargement of the spleen and great anæmia. In some cases, especially in animals where the malady runs a fairly rapid course, there are few changes to be discerned after death.

Dog No. 2.-This animal was inoculated subcutaneously with 1.5 cubic centimetres of the blood of Dog No. 1 Parasites appeared in the blood three days after inoculation and the animal died 15 days after inoculation.

Dog No.3.-Inoculated with the same dose and blood and at the same time as Dog No. 2. Parasites appeared two days, and the animal died 15 days, after inoculation. The spleen in this case was enormously enlarged and presented a peculiar raspberry colour which is by no means uncommon in this malady.

Dog No. 4.-Inoculated subcutaneously with five cubic centimetres of the blood of Calf No. 1. Parasites were seen sparingly until the twelfth day, after which they increased and remained numerous until death, which occurred on the nineteenth day.

Dog No.6.-The inoculations made into this dog are of particular interest. On June 26th, 1903, five cubic centimetres of the blood of a calf were injected subcutaneously. The calf had been inoculated ten months previously, had developed parasites, had suffered from the disease, had recovered, and had been since that time freanently reinoculated with virulent blood. No infection was found to follow in the dog inoculated with the blood of this calf and accordinglp it was again inoculated from the same calf on July 3rd, 1903. No infection followed. It was then inoculated with blood from another calf (which was then suffering from active infection). Eight days later parasites appeared in the blood in considerable numbers and augmented so rapidly that on the following morning the blood swarmed with them. The dog died suddenly on the ninth day.

Dog No. . - On Oct $16 \mathrm{~s}, 1903,0.5$ cubic centimetre of the heart blood of an infected rabbit was mixed with 50 cubic centimetres of serum taken from an immune calf (the former of the two mentioned) and the mixture was injected subcutaneously. On the 21 st a further inoculation of the same serum without infected blood was made. On the 22nd a few trypanosomes were seen in the blood and they remained numerous thereafter until death, which occurred on the eighteenth day.

Dog No. $8 .-0.5$ cubic centimetre of the blood of the same rabbit as was used in the preceding case was mixed with 50 cubic centimetres of serum from a horse which bad been highly immunised against South African horse sickness and the mixture was injected subcutaneously on Oct. 16th. On Oct. 21st parasites were seen in the blood and the dog died on the thirty.third day.

The foregoing experiments show the great variation which is commonly found with this class of disease and which is not entirely attributable either to the amount of dose or the mode of infection. For the purpose of comparison we may briefly indicate the periods between the moments of infection and death which bave resulted in our experiments in guinea-pigs, rabiits, and dogs with the tsetse fly and Mauritius trypanosomes respectively.

Manritius disease.-Eight dogs: $35,15,13,19,17,9,18$, and 33 days. Longest, 35 days ; shortest, 13 days; average, $19 \cdot 8$ days. Seven guinea-pigs: $66,49,59,51,10,30$, and 10 days. Longest, 66 days; shortest, 10 days; average $39 \cdot 3$ days. Eight rabbits : $53,59,55,2,32,53,18$ and 9 days. Longest, 59 days; shortest, 2 days ; average $35 \cdot 1$ days. The enormous rapidity of the evolution of the malady in the case of one animal which died two days after intraperitoneal injection is inexplicable and fallacy seems eliminated since the animal was a healthy one purchased locally for the experiment. Since, however, inoculations made with its blood into a guinea-pig and a rabbit were followed by long periods of infection in both cases, it is probable that the cause must be looked for in an unusual lack of resistance and possibly in the mode of inoculation by intraperitoneal injection.

Tsetse:fy dixease.-Two dogs : 10 and 11 days. Average, 105 days. Four rabbits : $15,26,21$, and 26 days. Longest, 26 days; shortest, 15 days; average, 22 days. Six guineapigs : $35,33,25,33,17$, and 17 days. Longest, 35 days ; shortest, 17 days ; average 26 days.

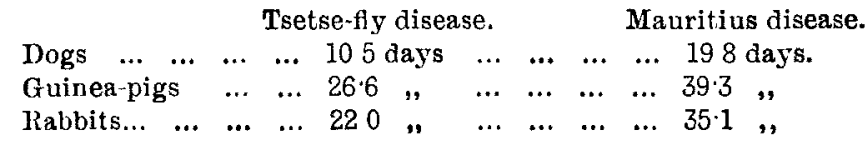

Thus for dogs, guinea-pigs, and rabbits the tsetse-fly disease appears to be lethal in less than two thirds of the time taken by the Mauritius disease.

The Mauritius disease was also inoculated into some other animals. 20 cubic centimetres of blood from a horse infected with the malady, and whose blood was swarming with parasites, were injected subcutaneously into a young male pig. No trypanosomes were ever found in the blood and during three months subsequent to inoculation the animal never showed the slightest illness but became exceedingly fat. We regret that no animals were injected with its blood and hence, in view of the observations we shall have to refer to in connexion with goats, it is impossible to conclude that infection did not occur. A pigeon was inoculated by subcutaneous injection in the thoracic region with 0.5 cubic centimetre from Dog No. 1. No trypanosomes were ever observed in the blood subsequently. A horse was inoculated by intravenous injection with 20 cubic centimetres of the blood of Dog No. 2. Parasites appeared in the blood in small numbers on the fourth day and were swarming in the blood by the seventh day. Between this period and the sixteenth day they were again few in numbers and the temperature had fallen somewhat. On the nineteenth day no trypanosomes were to be seen. The animal now showed great œdema of the sbeath which extended along the abtomen. From the twenty-first day parasites were exceedingly few until the thirty-first day, when the blood was once more swarming. The horse died on the following day. A calf (about 18 months old) was inoculated by intravenous injection with one cubic centimetre of the blood of a Mauritius dog on Sept. 4th, 1902. On the $8 \mathrm{th}, 9 \mathrm{th}, 11 \mathrm{th}$, and $12 \mathrm{th}$ of the same month parasites were present in the blood in considerable numbers. From the $14^{\mathrm{t} h}$ none were seen. On the $22 \mathrm{nd}$ the calf was reinoculated, by intravenous injection, with three cubic centimetres of the blood of Dog No. 1. No parasites were seen. On Oct. 8 th it was inoculated for the third time, receiving 25 cubic centimetres intravenously and 100 cubic centimetres subcutaneously of the blood of the horse already mentioned which swarmed with parasites. No parasites were seen. On Oct. 28 th a fourth inoculation was made 
when it received 50 cubic centimetres intravenously and 100 cubic centimetres subcutaneously of the blood of the infected horse. On Nov. 3rd a very few parasites were found in the blood and on the following day the blood was found to be swarming. Thereafter none were to be seen. On Nov. 15th a fifth inoculation was made, giving it 50 cubic centimetres intravenously and 100 cubic centimetres subcutaneously of virulent blood. No parasites were seen for nearly three weeks, when on Dec. 4th a few were seen and again on the 18 th and 24 th of the same month. On the 29 th they were numerous. A few were also seen on the following dates: Jan. 8th, 12th, 15tb, 21st, 22 nd, and $29 \mathrm{th}$, and Feb. 4th, 11th, and 12th, 1903. No more were seen until May 20th, when suddenly on, and only during, that day the blood was swarming. On no previous occasion had so many been seen, and the numbers were so great as to make the blood appear to consist almost entirely of parasites. On the subsequent day it was found that no parasites could be seen, nor have they ever been seen since that date in this animal. Four weeks later, and on numerous occasions at later dates, it was attempted to obtain infection with this calf's blood in dogs, guinea-pigs, and rats, but every attempt completely failed. The calf seemed therefore to have developed an active immunity, but the better to assure ourselves of this determined efforts were made to reinfect it and to obtain infection after these opera. tions. Thus on Nov. 2 nd, about six months after the last appearance of trypanosomes in its blood, it was inoculated with 20 cubic centimetres of the blood of an infected dog On Nov. 3rd and again on the 5 th white rats were inoculated with its blood. Neither of these, bowever, took infection but remained healtby. On Feb. 1st, 1904, we had fully assured ourselves that the animal had developed active immunity to the Mauritius disease, and as a control we injected 20 cubic centimetres of its blood into a rabbit which never became infected.

Experiment to determine whether an animal immunised to the Maurstius disease is also immune to the tsitse Hy disease.Immediately subsequently to this we inoculated the calf, now grown to a fair-sized ox, with 30 cubic centimetres (intravenously) of the blood of a dog infected with the tsetse-fly trypanosome. During 11 days subsequently to this we failed to observe any trypanosomes, but when the blood was used to inoculate a rabbit it proved virulent. The rabbit died 31 days after inoculation with the tsetse fly disease.

Regarding the failure to observe trypanosomes in the blood of the calf during 11 days after inoculation it may just be said that cattle do not always show trypanosomes in their blood within this time. Thus of two calves which were inoculated with tsetse-fly disease--one, which received five cubic centimetres of the blood of an infected dog, never showed trypanosomes in its blood during observations extending over several weeks. In the case of the other, which received 20 cubic centimetres of the blood of an infected rabbit, a few trypanosomes were found in its blood four days after inoculation but never again during ten months of observation. Here be it noted that in the tsetsefly regions of South Africa there is a popular belief that cattle attacked by the tsetse fly will show no signs of illness until the rains come, after which illness quickly develops. It would appear, however, that the strain of tsetse-fly disease with which we were working possessed a low order of virulence for cattle. At the same time it is noteworthy that inoculation of a Mauritius-immune ox with tsetse-fly disease showed that the animal was not immune to the latter malady. But as immunity to both trypanosomes was very soon established in the Mauritius-immune ox it is possible that the Mauritius disease assisted in establishing active immunity towards the tsetse-fly disease, although it could not do so completely of itself. It would also seem that for the previous reasons we are bound to regard the two trypanosomes as specifically distinct.

This, the first recorded demonstration of the fact that an active and a more or less permanent immunity towards a pathogenic trypanosome can be experimentally produced in animals, was first communicated to the Cape Government in the report of the director of the Colonial Bacteriological Institute in 1903 It is amply confirmed by the work of Laveran and Mesnil on smaller animals and by the results obtained in oxen at Mauritius and published in the report of the director of the health department of the island for 1904.

In the report of the superintendent of the Government laboratories of the Philippine Islands for $1903 \mathrm{Dr}$. Musgrave and Dr. Cleyg consider that the urypanusulua Evalisn and try panosoma Brucei are identical, and hence that the latter, being of later discovery, should have no place in our nomenclature. They say: "The proof demanded is that they are different parasites and, until the proof is furnished, writers, in our opinion, are permitted to consider the trypanosomiasis of horses and a number of other animals as being due to an infection with trypanosoma Evansii.'

We consider now that ample proof is furnished as to the specificity of trypanosomes the morphology and even pathogenic action of which may nevertheless be exceedingly similar.

Towards the end of $19 \mathrm{C} 4$ by an unfortunate accident on stock of infected small animals ran out and our supply of infecting material was lost. With a view to re establishing it we tried by every mode of inoculation possible to procure infection from cattle immunised to the Mauritius and tsetsefly diseases respectively. All attempts in even enormous doses absolutely failed, and in this way was established, if that were indeed necessary, a striking demonstration as to the possibility of immunising cattle against these trypanosomes.

Dr. Lorans, medical director at Mauritius, also reports that cattle inoculated there for one of us (A. E.) in 1902 are still alive and that their blood is not infective to susceptible animals.

In the case of goats, experimentally infected with the Mauritius disease, some most interesting features were dis. closed. Five Angora goats have been inoculated at different dates, of which all have died from the disease. The periods during which they survived were as follows: $96,55,43,33$, and 52 days. The longest period was 96 dajs and the shortest 33 days, while the average was $55 \cdot 8$ days. In the case of the second goat, which received 20 cubic centimetres intravenously and 50 cubic centimetres subcutaneously of the blood of a horse in which trypanosomes swarmed, one or two immobile bodies of questionable nature were seen on examination of the blood two days later. With this exception the most painstaking examination has invariably failed to. detect the presence of any parasites in the blood or other fluids of these animals. Lumbar puncture was also resorted to in order to determine whether some parasites might not. be found there, but here also the result of examination proved negative. One goat was killed during the late stage of its illness and a searching examination was then made by sections and smears of most organs-viz., the lungs, the heart, the liver, the kidneys, the brain, the spinal cord, and glands and various of its fluids. Examination, however, failed to show anything which could be considered as of the nature of trypanosomes. In the experiment only Angora goats were used and it would be interesting to determine whether in other species or in sheep similar results would follow the inoculation of the Mauritius disease. In the case of tsetse-fly disease Bradford and Primmer state with regard to experimental inoculation that " the goat (species not defined) shows very little sign, and the organism is not found abundantly in the blood, but the animal gets cedema of the genitals, and the eyes becomesomewhat opaque. It dies in about two months after inoculation, with paralysis. The spleen is not enlarged; the nasal mucous membrane becomes swollen and interferes with breathing." In the case of the goats used by us the principal sign during life was the progressive emaciation which supervened and which proceeded to so great an extent that the animals became veritable skeletons before death overtook them. The mucous membrane became swollen, the breathing was obviously disturbed, and the opacity of the eyes was always most marked. Towards the end of the period the animals were invariably drowsy and scarcely moved even to feed. Indeed, the picture thus presented might be considered as analogous to the late stages of human trypanosomiasis when the condition of "sleeping" sickness" has been established.

The remarkable and even rapid disappearance of trypanosomes from the blood of immunised animals into which large doses of these parasites have been injected is in all probability more or less accounted for by phagocytosis. We have seen ourselves, on the stage of the microscope, a white corpuscle attack and devour two trypanosomes in rapid succession. In this case the two parasites made up a. bulk which seemed greatly superior to that of the white corpuscle itself, yet, remarkable to relate, very little evidence of increased bulk in the white corpuscles was noticed. It is therefore very probable that a cytolytic action is concerned in the process. 
By the kindness of Dr. Lorans we are enabled to show a harboured by bovines and that, under favourable conditions, chart illustrating the progress of the disease in Mauritius from Jaly, 1902, until the end of 1904. The figures prior to July, 1902, are not, unfortunately, available.

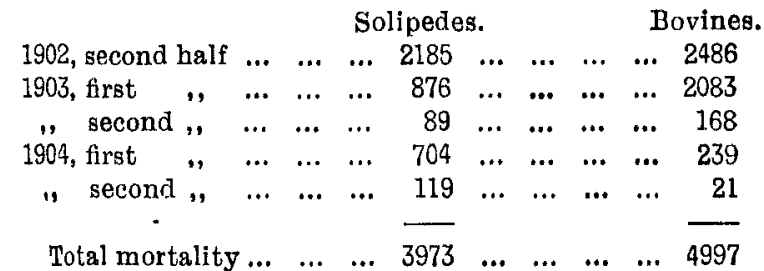
cattle possess a natural resistance to the disease, since the animals experimented upon have been put to work and are, with the exception of one which, it is believed, died of accidental causes, in the best condition. Two bullocks treated in the manner indicated by Dr. Edington have given on two occasions negative results on rabbits, thereby apparently confirming his opinion that the injection of virulent surra blood may produce tolerance and recovery after a time." It may be gathered from this interesting report that Dr. Lorans is definitely of opinion that trypanosomiasis in Mauritius has become actually less virulent for bovines. Unfortunately, however, the animals which are continually being imported into Mauritius include mules as well as oxen, and, since in the former little or no resistance is found, the importation of these animals more than anything else tends to extend

Commenting on the figures submitted, the director of the health department states: "In 1904 the epizootic has been less viralent in both equines and bovines, but the improvement is specially noticeable in the cases of the latter.

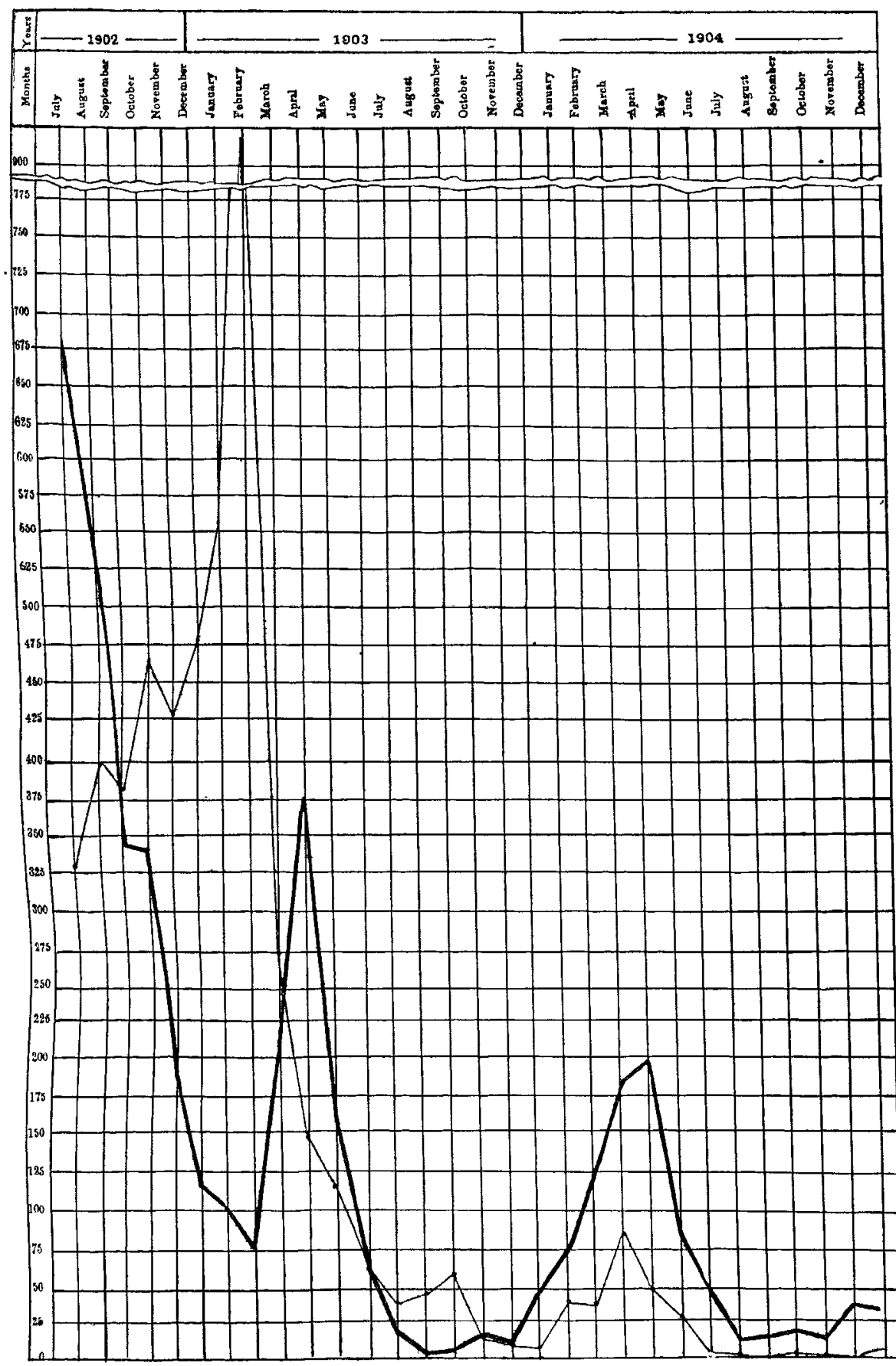

Chart showing the monthly number of bovines and solipedes which died from surra or were slaughtered on account of surra during the years $19(2,1903$, and 1904. Thin line=bovines. Thick line=solipedes.

Allowances being made for the altered conditions of transport on the plantations and the variations in the number of animals yearly imported into the island, it seems possible to infer from the chart and other known facts that 'surra' has assumed a less virulent form among the cattle than cquines, for the decrease in the number of deaths recorded for cattle in 1903 and 1904 is much more marked than the correspond. ing figure for equines during the same periods. The latter do not resist the disease, while the former frequently recover naturally so long as they are placed under favourable conditions. Experiments have shown that the disease can be
Regarding the evolution of trypanosomiasis from the points of view of experimental inoculation and observation in naturally infected animals, it seems probable that the virulence of the infection in a specific locality may become altered in type and tend to assume a latent form in animals in which the disease usually runs a chronic course.

As regards the infection harboured by certain big game, it seems probable that it follows the line which has been observed in cattle, and hence, while younger animals may be in active infection for some months, probably the older ones become immune.

We regard the observations made by us concerning goats as of considerable import since in this animal, at least, the disease can exist in a form not readily recognised by blood examination; when, indeed, trypanosomes cannot be seen although the inoculation of their blood to susceptible animals is followed by the disease and appearance of the characteristic trypanosomes. Obviously, therefore, what has obtained in goats for this species of trypanosome, for aught that we yet know, might find its analogue in other animals for other species of trypanosomes.

The evidence shown by our experiments that it is possible to immunise cattle against the diseases caused by the tsetse-fly and Mauritius trypanosomes is of value since it establishes the possibility of preparing cattle to act as means of transport in infected areas.

It would be of great interest to determine the particulate form of the parasite in goats when trypanosomes are not evident. We have made one experiment of injecting filtered goat's blood into a susceptible animal but the result was negative. This experiment is worthy of further repetition.

Our experiments with serum derived from immunised animals do not warrant us in holding out any hopes that much practical utility is to be expected from this material.

\section{NuRses' Missionary League.-} Two valedictory meetivgs will be held in connexion with the Nurses' Missionary League at University Hall, Gordon-square, London, W.C., on Tuesday, Oct. 8th. An afternoon conversazione will be held from 2.30 to 6 P.M., and an evening meeting from 7 to 9 o'clock. These meetings have been specially arranged to bid God-speed to ejght members of the Nurses' Missionary League who will leave England this autumn for foreign lands-five going to India and three to China. All friends interested in nursirg and hospital life, whether at hcme or abroad, are invited to attend. Inquiries should be addressed to the honorary secretary, Miss Ricbardson, 52, Lower Sloane-street, London, S.W.

The Bristol Medico-Chirurgical Society will meet at the medical library, University College, Bristol, on Wednesday, Oct. 9th, at 8 P M when the president, Dr. Henry Waldo, will give the inaugural address. 